\section{Quick as a Flash: \\ Ultrashort Laser Sources}

\section{Sandro De Silvestri and Orazio Svelto Milan Polytechnic, Italy}

The authors review the most significant steps in the generation of ultrashort light pulses and highlight the potential of recently developed laser sources for the study of light-matter interactions at unprecedented power levels

Short light pulses have been used as a means of freezing the motion of fast occurring events since the invention of flash-photography in the 19th century. Thanks to the discovery of the laser in 1960 , researchers have been able to develop a detailed understanding of many optically-initiated processes that occur on nanosecond, picosecond and femtosecond time-scales. During the last two decades the study of previously unresolved fast processes in physics, chemistry and biology has shed new light on the dynamic properties of matter.

The first significant step in the generation of ultrashort laser pulses came in 1966, when pulses of less than locking in phase the longitudinal modes of a Nd:glass laser, using a fast saturable absorber. From then on, this technique (named passive mode-locking) provided the basis and the stimulus for technological devel-

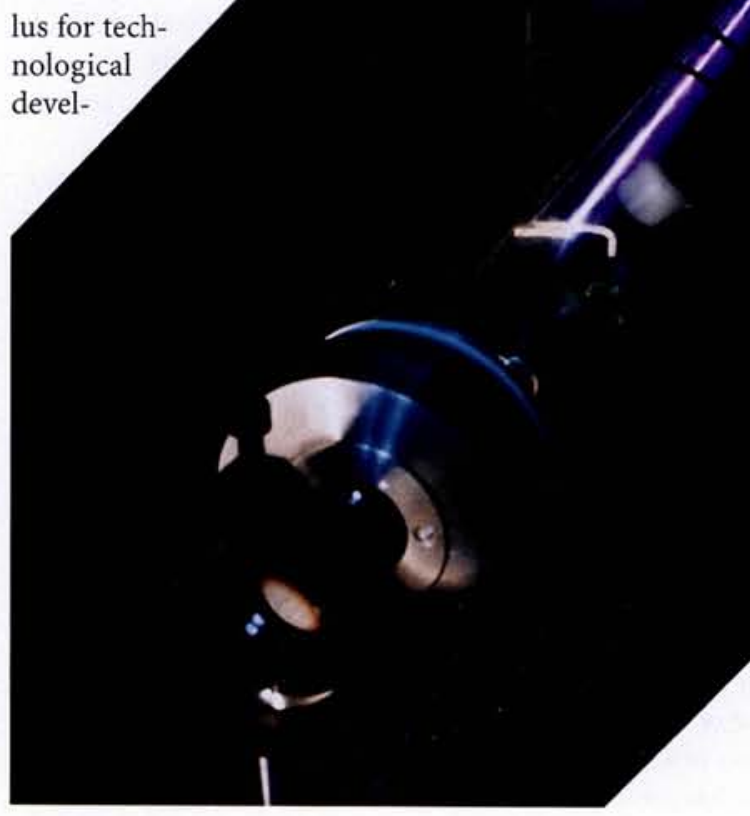
10 picoseconds were obtained by opments in the field of ultrashort pulse generation. By using materials with high gain over a broad bandwidth such as laser dyes, light pulses of 1.5 ps duration were generated in 1972 and as short as $90 \mathrm{fs}$ in 1981 in a ring dye laser. Femtosecond mode-locked dye lasers, whose pulse energy was raised by cascading amplification stages, became the basic tools for ultrafast spectroscopy in all branches of science during the 1980 s.

In 1991 an exciting result was obtained at the University of St. Andrews: the selfmodelocking of a Ti:Sapphire laser.

Indeed, a new mode-locking technique was discovered: a time-dependent lens, induced in active material by the intensity dependence of the refractive index (Kerr effect: see box), produces, on a pulse travelling in the cavity, more losses on the wings than on the peak if an aperture is placed in the resonator. Under these conditions, a pulse shortening mechanism acts at each round trip. The Kerr-lens-modelocking (KLM) technique revolutionized the field of ultrashort pulse generation and led to the renaissance of solid state lasers. The average power was raised from the 10 milliwatt level typical of mode-locked dye lasers to about $1 \mathrm{~W}$.

Nowadays Ti:Sapphire laser oscillators provide pulses of sub-10 fs duration. A key feature used in obtaining such short pulses is the ability to control group velocity dispersion in the laser cavity. A great technological improvement was achieved by incorporating in the cavity chirped (see box) mirrors, characterized by layers of different thicknesses in order to change group delay over a large bandwidth. Since the KLM technique is not limited to a particular wavelength, other active media containing $\mathrm{Cr}^{3+}$ or $\mathrm{Cr}^{4+}$ ions in different host matrices can provide pulses with a duration varying from $20 \mathrm{fs}$ to $100 \mathrm{fs}$ in the wavelength range $800-1500 \mathrm{~nm}$.

The pulse energy of femtosecond laser oscillators usually ranges from hundreds of picojoules to a few nanojoules. Until 1985 the amplification of ultrashort laser pulses was restricted to dye amplifying media due to their ultra-broadband gain bandwidth and low saturation energy. This situation drastically changed with the introduction of chirped pulse amplification (CPA), which enabled the amplifica-

Below Measured (solid line) and calculated (dots) trace of the pulses obtained by pulse compression using a hollow fibre filled with krypton Left the pulse itself 


\section{The Kerr Effect \\ The optical Kerr effect is the change in the refractive index of a medium due to an electric field. In the present context, the electric field is due to the light wave itself, and the refractive index $n$ varies as $n=n_{0}+n_{2} I$ where $n_{0}$ is the usual or zero- intensity value of $n$, while $n_{2}$ is the Kerr contribution and $\mathrm{I}$ is the intensity of the laser beam}

tion of ultrashort pulses to very high power levels by using solid-state amplifying media with better energy storage capability (larger saturation energy). In the CPA technique a low energy pulse is first passed through a dispersive optical delay line. Since either energy or bandwidth are preserved, the output pulses are lengthened in time and chirped, ie the carrier frequency changes within the envelope of the pulse. The reduction in pulse peak power, due to stretching the pulse out in time, is essential to avoid any optical damage during amplification. After amplification the pulse is recompressed in time near to its original duration by using an optical delay line with opposite dispersive effects.

In recent years, there has been significant progress in the developments of CPA amplifiers. Advanced table-top Ti:Sapphire amplifiers, seeded by 10 fs laser oscillators, able to generate 20-30 fs pulses with tens of terawatt peak power at repetition rates in the $10 \mathrm{~Hz}$ region, have been built at the University of San Diego in USA, Ecole National de Techniques Avancées in France, the University of Tokyo and the Japan Atomic Energy Research Institute in Kansai. In the kiloHertz regime laser systems now provide pulses of about 20-30 fs with $200 \mathrm{GW}$ peak power. Extensions to the terawatt level of high repetition rate lasers is currently under development at the University of Bordeaux in France and at the University of Michigan in USA with the construction of $1 \mathrm{kHz}, 1$ TW laser facilities. The concept of CPA, implemented on existing large laser systems built for laser fusion at Lawrence Livermore National Laboratory and Los Alamos National Laboratory in the US, the Commissariat a l'Energie Atomique in France, the Rutherford Appleton Laboratory in the UK and the Institute of Laser Engineering in Osaka, Japan, has transformed nanosecond lasers down to the sub-picosecond time domain, with at the same time an increase in the peak power by orders of magnitude up to 0.1 to 1 petawatts.

A valuable technique for pulse shorten- ing is represented by pulse compression outside the laser cavity. Light pulses are first spectrally broadened by propagation in a non-linear guided structure and then temporally compressed through a suitable optical dispersive delay line. Using singlemode optical fibres with a prism-grating compressor, pulses of 6 fs duration were obtained in 1987 from an amplified modelocked dye laser and more recently of sub5 fs duration from a cavity dumped Ti:Sapphire laser. However, single-mode fibres limit the input pulse energy to a few nanojoules due to possible material damage and unwanted non-linear effects. A powerful pulse compression technique based on spectral broadening in a hollow fibre filled with noble gases has demonstrated the capability of handling high energy pulses ( $\mathrm{mJ}$ range). This technique presents the advantage of a guiding element with a large diameter mode and of a fast nonlinear medium with high damage threshold. Combination of the hollow fibre technique with a dispersive delay line, which includes chirped mirrors, has recently led to the compression of $20 \mathrm{fs}$ pulses down to $4.5 \mathrm{fs}$ at multigigawatt peak power level. These pulses are the shortest generated to date at this power level.

The application of high peak power lasers to several problems in physics and chemistry has been as rapid as the development of laser technology. Over the past ten years laser intensities have increased by more than four orders of magnitude, reaching values of $10^{20} \mathrm{~W} / \mathrm{cm}^{2}$. At intensities greater than $10^{15} \mathrm{~W} / \mathrm{cm}^{2}$ one leaves the perturbative approach of non-linear optics to penetrate into a regime characterized by electric fields much larger than the coulomb field. Under these conditions the quiver electron velocity in the laser field is close to the speed of light giving rise to new laser-matter interactions. New physics and applications now become accessible such as high order harmonic generation, non-linear relativistic effects, laser acceleration and thermonuclear ignition. The most recent developments in high order harmonic generation can be considered as a typical example of the border line between conventional non-linear optics and new physics.

Beyond about $10^{14} \mathrm{~W} / \mathrm{cm}^{2}$, gas atoms exposed to intense laser radiation can emit coherent harmonic radiation with photon energies much higher than the atomic binding energy. Since the electric field of the laser is now approaching that of the Coulomb field, the bound electron can tunnel through the Coulomb barrier lowered by the high intensity laser field. The quasifree electron acquires kinetic energy from the laser field and depending upon the phase of the laser field, after some half optical cycle, can return to its parent ion and recombine to the ground state. The emitted photon has the energy of the atomic ionization potential plus the kinetic energy acquired by the laser field. The process, repeated periodically in the field of the driving pulse, gives rise to the emission of a coherent beam of harmonics of the incident radiation. The highest harmonic photon energy is dictated by the maximum laser intensity that neutral atoms can be exposed to before they are depleted by ionization. This critical intensity turns out to be higher for driving pulses of shorter duration. Recent results have shown that with $26 \mathrm{fs}$ pulses at $780 \mathrm{~nm}$, wavelengths as short as $2.7 \mathrm{~nm}(460 \mathrm{eV})$ can be generated in helium. Using quasi-single cycle optical pulses of 5 fs duration, a continuum spectrum extending below $4.3 \mathrm{~nm}$ has been observed, making also feasible the possibility of generating attosecond pulses $\left(10^{-18} \mathrm{~s}\right)$ in this spectral region for the first time. The great attraction of high harmonic generation is that it is a simple and compact source for coherent soft X-rays. A major application of this source is for high contrast biological spectroscopy in the water window (2.33-4.37 nm), where carbon-containing biological objects absorb radiation efficiently but water is transparent. In addition, this source offers the possibility of extending non-linear optics into the $\mathrm{X}$-ray regime and studying atomic processes with potentially attosecond time resolution.

In conclusion, the development of ultrashort laser sources during the last two decades based on CPA, solid state technology and dispersion control tools has allowed the building of table top systems able to provide intensity levels up to $10^{20} \mathrm{~W} / \mathrm{cm}^{2}$. Small scale, high performance lasers will continue to attract further interest from physicists for laboratory-sized experiments.

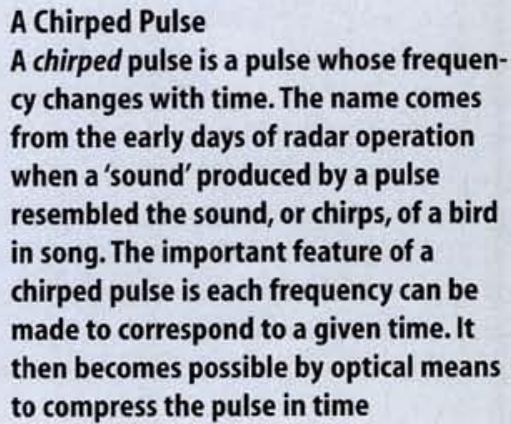

